\title{
Atrial and Ventricular Deformation Analysis in Normal Fetal Hearts Using Two-Dimensional Speckle Tracking Echocardiography
}

\author{
Markus Meister $^{\mathrm{a}}$ Roland Axt-Fliedner ${ }^{\mathrm{a}}$ Oliver Graupner ${ }^{\mathrm{b}}$ Viktoria Kuhn ${ }^{\mathrm{a}}$ \\ Aline Wolter ${ }^{\mathrm{a}}$ Malena Götte $^{\mathrm{a}}$ Christian Enzensberger $^{\mathrm{a}}$ \\ aDepartment of OB/GYN, Division of Prenatal Medicine, University Hospital UKGM, Justus-Liebig University, \\ Giessen, Germany; 'D Department of OB/GYN, University Hospital Rechts der Isar, Technical University of Munich, \\ Munich, Germany
}

\section{Keywords}

Speckle tracking echocardiography - Atrial deformation · Atrial and ventricular strain - Myocardial function - Systolic strain

\section{Abstract}

Objective: Two-dimensional speckle tracking echocardiography (2D-STE)-based strain values of the left and the right ventricle have been established; however, less is known about atrial deformation. The aim of our study was to assess both atrial strain and ventricular strain using 2D-STE in a cardiac 4-chamber view and to investigate the effect of possible influencing factors such as gestational age. Methods: Fetal echocardiography was performed on a Toshiba Aplio 500 ultrasound system. Based on an apical or basal 4-chamber view of the fetal heart, left and right ventricular longitudinal peak systolic strain (LVLPSS and RVLPSS) as well as left and right atrial longitudinal peak systolic strain (LALPSS and RALPSS) were assessed by 2D-STE. Results: A total of 101 healthy fetuses were included. The mean gestational age (GA) was $26.0 \pm 5.6$ weeks. GA was significantly positively correlated ( $p<0.05$ ) with LVLPSS and RVLPSS and significantly negatively correlated $(p<0.05)$ with LALPSS and
RALPSS. The mean values for LVLPSS and RVLPSS were $-17.44 \pm 2.29 \%$ and $-16.89 \pm 1.72 \%$. The mean values for LALPSS and RALPSS were $34.09 \pm 4.17 \%$ and $35.36 \pm 2.90 \%$. Conclusion: Ventricular and atrial deformation analysis in 2D-STE was technically feasible and showed comparable values to current data. For future research on myocardial function (MF) of the fetus, considering GA as an influencing factor for deformation analysis seems to be adequate. Especially, atrial deformation analysis allows the assessment of diastolic myocardial function. Further research needs to clarify the clinical meaning of these myocardial deformation indices in fetuses at risk.

(C) 2020 The Author(s)

Published by S. Karger AG, Basel

\section{Introduction}

Two-dimensional speckle tracking echocardiography (2D-STE) is an angle-independent technique to quantify myocardial function (MF) of the fetus. 2D-STE has been applied in several pathological conditions such as twinto-twin-transfusion syndrome, congenital diaphragmatic hernia, maternal diabetes, or congenital heart disease [1-4].

$\begin{array}{ll}\text { karger@karger.com } & \begin{array}{l}\text { (c) 2020 The Author(s) } \\ \text { Published by S. Karger AG, Basel }\end{array} \\ \text { www.karger.com/fdt } & \text { This is an Open Access article licensed under the Creative Commons } \\ \text { Karger } & \begin{array}{l}\text { Attribution-NonCommercial-4.0 International License (CC BY-NC) } \\ \text { (http://www.karger.com/Services/OpenAccessLicense), applicable to } \\ \text { the online version of the article only. Usage and distribution for com- } \\ \text { mercial purposes requires written permission. }\end{array}\end{array}$

Christian Enzensberger

Universitätsklinikum Giessen Marburg

Klinikstr. 33, DE-35392 Giessen (Germany)

cenzensberger@googlemail.com 


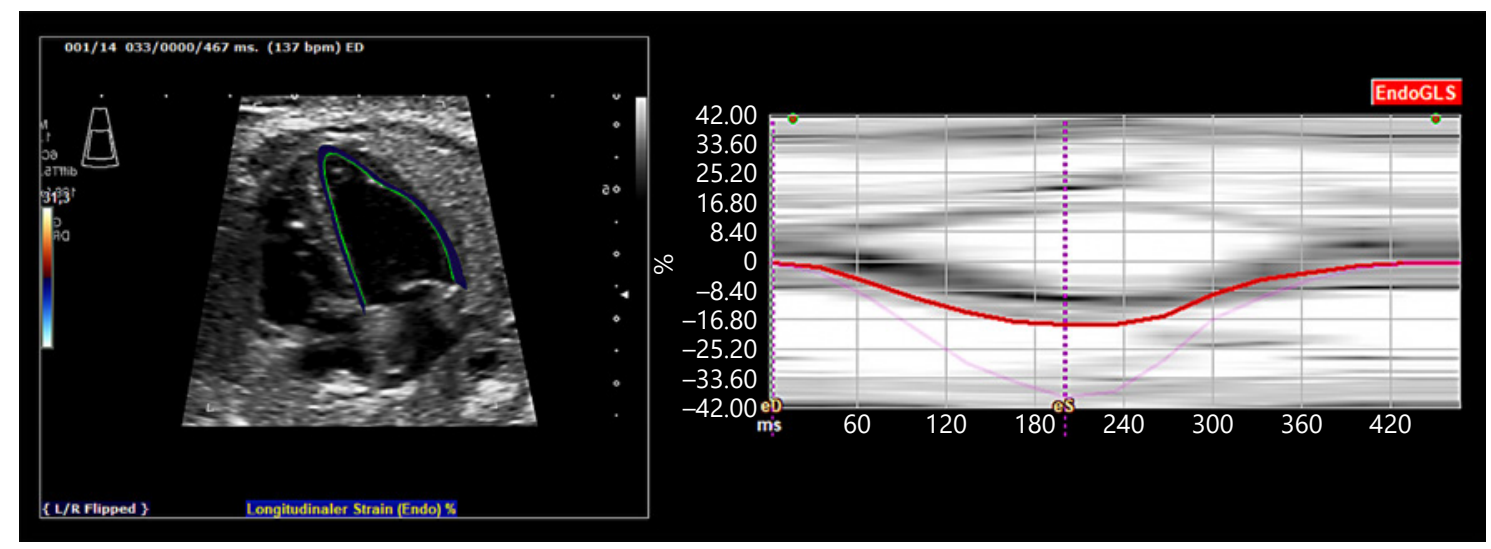

Fig. 1. Ventricular strain analysis. Left: $4 \mathrm{CV}$ of the fetal heart with myocardial border tracking of the left ventricle. Right: ventricular global longitudinal peak systolic strain measurement within 1 cardiac cycle. $4 \mathrm{CV}$, 4-chamber view.

Recent evaluation of deformation values by 2D-STE has gained greater acceptance, as it has shown to be feasible and reproducible for the assessment of both right ventricular (RV) and left ventricular (LV) performance in the fetus [5-12]. However, reported ventricular strain values for mean LV and RV global longitudinal peak systolic strain (LPSS) show a widespread ranging $[8,10,13-$ 17]. Our own work revealed that the variability in image acquisition, optimal temporal resolution, and use of different ultrasound software packages are important influencing factors and possible reasons for this divergent strain values [10-12]. On the other hand, data on fetal myocardial deformation properties of the left atrium (LA) and right atrium (RA) are sparse [18, 19]. However, current data in adult cardiology shows feasibility and acceptable reproducibility of atrial 2D-STE. Furthermore, these data illustrate the potential of atrial deformation parameters, which may act as predictors for the necessity of therapeutic interventions in adult cardiology [20]. Besides already investigated potential function of 2D-STE to distinguish between healthy fetuses and fetuses with congenital heart diseases (CHD), the function of myocardial strain as a predictor for the success of cardiosurgical interventions may be a potential usage for 2D-STE [21,22]. To enable future research in these areas, the aim of our study was to evaluate atrial and ventricular MF using 2DSTE in normal fetal cardiac 4-chamber view (4CV), which is a common standard in basic sonographic screening, and test reproducibility of our collected deformation values. In addition, the possible influence of gestational age (GA) and fetal heart rate (FHR) on atrial and ventricular LPSS analysis should be assessed. Especially, atrial defor- mation analysis allows the assessment of diastolic MF and could give additional insights into the pathophysiology of CHD in this way.

\section{Methods}

\section{Study Population}

We retrospectively analyzed prospectively acquired data on healthy fetuses between the 16th and 39th week of gestation selected from women referred for fetal echocardiography from April 2014 to March 2016. Some of these fetuses were reported in previous studies [10-12].

All patients underwent a morphological examination of the fetal heart in order to exclude structural anomalies. Cases with the evidence of chromosomal anomalies, twin pregnancies, or other conditions with possible effect on fetal hemodynamics such as pre-eclampsia, preterm labor, or endocrine disorders (e.g., thyroid disease and maternal diabetes) were excluded from analysis. Postnatally, a complete physical examination was performed to assess the clinical status of the neonates. The institutional review board approved this study. Informed written consent was obtained from all participants.

\section{Echocardiography}

According to our previous studies [10,12], fetal echocardiography was performed standardized by a highly experienced operator on a Toshiba Aplio 500 ultrasound system (Toshiba Medical Systems Corporation, Otawara, Tochigi, Japan) with a 1-5 MHz curved array probe (PVT $375 \mathrm{BT}$ ). Based on practical knowledge of previous data acquisitions, B-mode image depth was reduced, sector width was adjusted, and fetal as well as maternal movements were avoided to guarantee high-quality image acquisition with the aim to display every cardiac cavity in their full size. To perform offline strain analysis, a minimum of 3 cardiac cycles were recorded in preferably apical or basal $4 \mathrm{CV}$. These $\mathrm{B}$-mode cine loops were digitally stored in a standard format called Digital Imaging and Communications in Medicine (DICOM FR [60 fps]). 


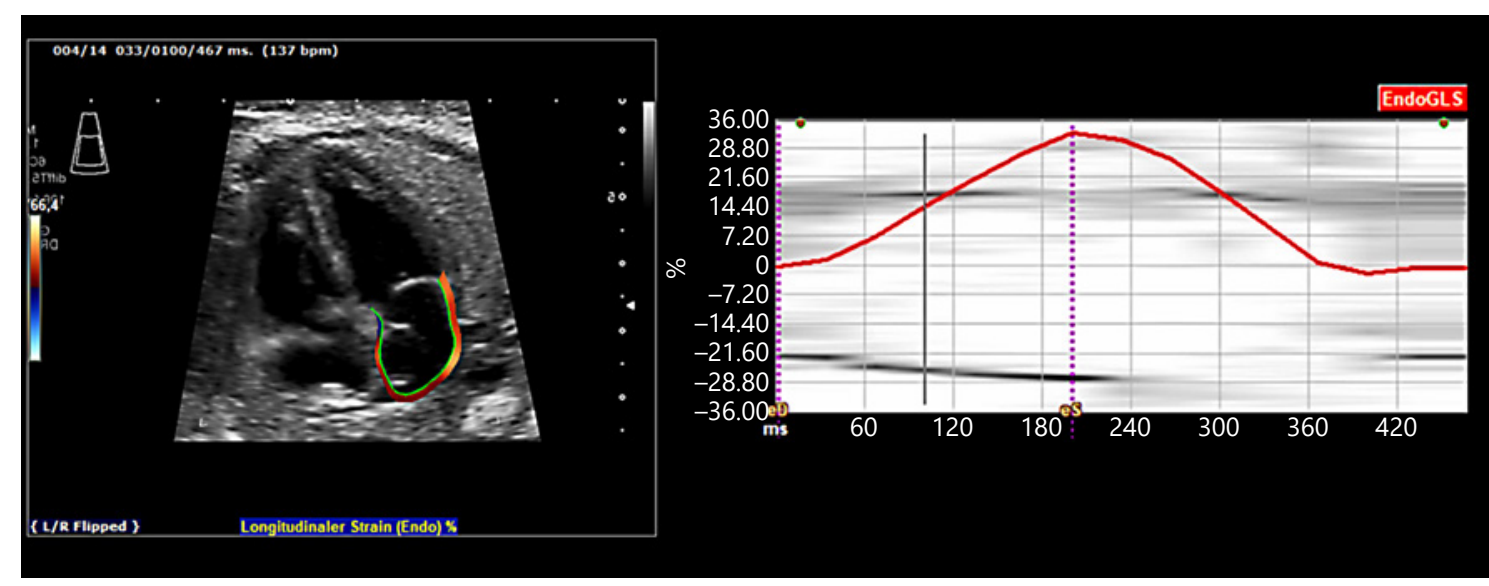

Fig. 2. Atrial strain analysis. Left: $4 \mathrm{CV}$ of the fetal heart with myocardial border tracking of the left atrium. Right: atrial global longitudinal peak systolic strain measurement within 1 cardiac cycle. 4CV, 4-chamber view.

Table 1. Study group with corresponding number of included $4 \mathrm{CV}$, mean, standard deviation, minimum, maximum, and 95\% confidence interval for mean of GA, FHR, and LPSS of all cardiac cavities and LV/RV ratio

\begin{tabular}{|c|c|c|c|c|c|c|c|}
\hline & GA & FHR & LVLPSS & RVLPSS & LALPSS & RALPSS & $\begin{array}{l}\mathrm{LV} / \mathrm{RV} \\
\text { ratio }\end{array}$ \\
\hline Total, $N$ & 101 & 101 & 101 & 101 & 101 & 101 & 101 \\
\hline Valid & 101 & 101 & 101 & 101 & 86 & 94 & 100 \\
\hline Missing & 0 & 0 & 0 & 0 & 15 & 7 & 1 \\
\hline Mean & 26.00 & 143.80 & -17.44 & -16.89 & 34.09 & 35.36 & 1.03 \\
\hline $\mathrm{SD}$ & 5.62 & 11.35 & 2.29 & 1.72 & 4.17 & 2.90 & 0.15 \\
\hline Minimum & 16.29 & 112 & -23.88 & -21.38 & 24.13 & 30.12 & 0.76 \\
\hline Maximum & 38.57 & 170 & -12.28 & -11.21 & 42.09 & 42.58 & 1.41 \\
\hline \multicolumn{8}{|c|}{$95 \%$ confidence interval for mean } \\
\hline Lower bound & 24.89 & 141.56 & -17.90 & -17.23 & 33.20 & 34.77 & 1.01 \\
\hline Upper bound & 27.11 & 146.04 & -16.99 & -16.55 & 34.99 & 35.96 & 1.06 \\
\hline
\end{tabular}

GA, gestational age; FHR, fetal heart rate; LV, left ventricular; RV, right ventricular; LA, left atrial; RA, left atrial; LPSS, global longitudinal peak systolic strain; LV/RV ratio, left and right ventricular LPSS ratio; 4CV, 4-chamber view.

Two-Dimensional Speckle Tracking Technology

Speckle tracking is an application of the 2D-Cardiac Performance Analysis ${ }^{\circledR}$ (2D-CPA) technology to ultrasound cine data to detect motion of the fetal heart. This method does not make use of Doppler information, so there is no Doppler angle dependency $[12,23]$.

\section{Strain Analysis}

Strain analysis was performed with the offline STE Software "TomTec Image Arena" (TomTec Imaging Systems GmbH, Unterschleissheim, Germany) by 2 trained investigators. One fetal heart cycle was identified and selected by anatomical M-mode using the closure of the mitral and tricuspidal valve as orientation for determinination of the end systole. Via three-point analysis, the operator set endocardial borders of every cardiac cavity. For this purpose, we set 1 point at each approach of the valves in the chamber and 1 point at the top of the chamber to be analyzed. Atrial and ventricular strain analysis varies in the location of the apex point (Figs. 1, 2). Once the points are defined, the software is able to detect the borders of the selected heart chamber automatically and performs a complete strain analysis. This analysis is based on the 2D-CPA technology, which in turn is based on the hierarchical algorithm at multiple scales and 1D and 2D feature tracking. Left and right ventricular longitudinal peak systolic strain (LVLPSS and RVLPSS) as well as left and right atrial longitudinal peak systolic strain (LALPSS and RALPSS) are displayed graphically and numerically. In consequence of different contraction directions related to the valve level, atrial LPSS is positive and ventricular LPSS is negative. Absolute values reflect the magnitude of deformation irrespective of its direction. To detect possible differences in ventricular MF between the single ventricles, LV/RV ratio was formed [10-12]. The FHR was determined 


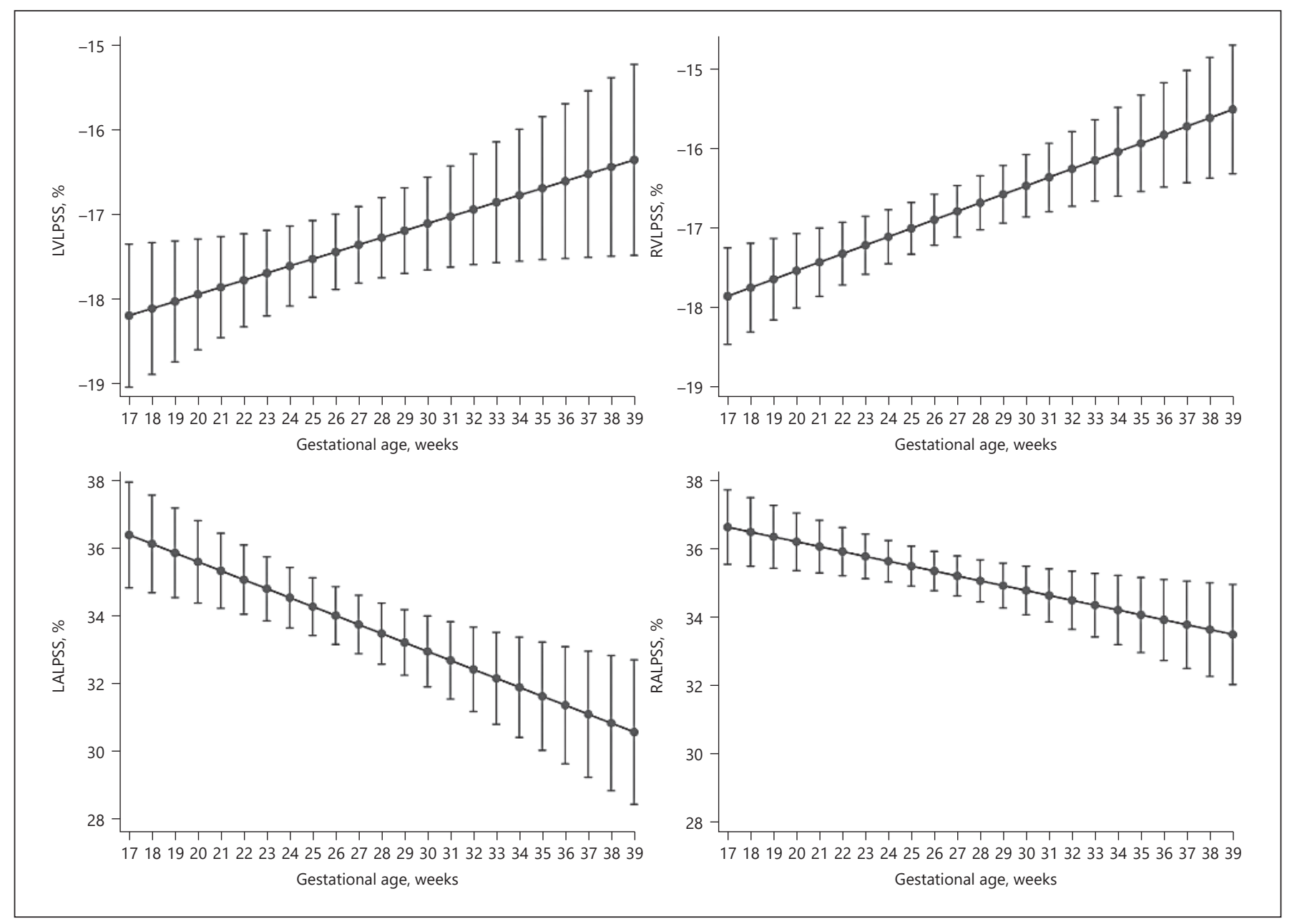

Fig. 3. Linear regression analysis of LPSS and GA with 95\% confidence interval. LV, left ventricular; RV, right ventricular; LA, left atrial; RA, right atrial; LPSS, global longitudinal peak systolic strain; GA, gestational age.

on the basis of one heart cycle duration, and out of this, frames per cycle $(\mathrm{fpc})$ were calculated. Tracking inaccuracy had to be corrected by the operator. How to perform a strain analysis in cardiac 4CV and handle tracking inaccuracies is shown in see online suppl. videoclip 1; for all online suppl. material, see www. karger.com/doi/10.1159/000508881. Nonsatisfactory strain analyses were excluded from final analysis, and the resulting dropout rate will be reported.

\section{Statistical Analysis}

IBM SPSS Statistics (Version 25.0 for Windows) and STATA (Version 15.1 for Windows) were used for statistical analysis. Using linear regression analysis, we observed the dependencies between the regressor $\mathrm{X}=\mathrm{GA}$ and the dependent variable $\mathrm{Y}=\mathrm{LPSS}$. Unstandardized regression coefficient $b$ is reported. Following the suggestion of Alan C. Acock, observations with standardized residuals larger than $|2.58|$ can be expected in less than $1 \%$ of the observations by chance and are defined as outliers [24]. In the following evaluation, a model without outliers and resulting dropout rate is reported. In case of significant association between GA and
LPSS, GA-dependent marginal means for LPSS are reported. To allow the comparison of our data with future examined strain values, we show the regression formula for every correlation. Analogously to Kurmanavicius et al. [25], from out of here Z-scores can be calculated using the formula: $Z=[\mathrm{X}-\mathrm{M}(\mathrm{GA})] / \mathrm{SD}(\mathrm{GA})$. To detect possible associations between the single LPSS values of different cardiac cavities, which may be used as indicators for more detailed clinical examination, Pearson correlation analysis was performed. Exemplary for LVLPSS, we analyzed the influence of the mediator variable $\mathrm{M}=\mathrm{FHR}$ on the dependency between $\mathrm{Y}=$ LVLPSS and $\mathrm{X}=\mathrm{GA}$ through mediation analysis, using the PROCESS 3.1 module for SPSS by Hayes [26].

All accomplished statistical tests were performed with a significance level of 0.05 . The data were validated by using double data entry. Descriptive statistics are presented as mean \pm 1 SD.

Twenty randomly chosen patients were used for calculating interrater reliability and 101 patients were used for calculating intrarater reliability with an interval longer than 8 weeks after the 1st measurement to limit recall bias. Reliability was examined with Bland-Altman analysis. 
Table 2. Marginal mean values for LPSS of all cardiac cavities for the 20th, 26th, 32nd and 38th week of gestation with $95 \%$ confidence interval

\begin{tabular}{llll}
\hline GA, week & \multirow{2}{*}{$\begin{array}{l}\text { Marginal } \\
\text { mean }\end{array}$} & \multicolumn{2}{l}{$95 \%$ confidence interval } \\
\cline { 3 - 4 } & & lower bound & upper bound \\
\hline LVLPSS, \% & & & \\
20th & -17.95 & -18.6 & -17.29 \\
26th & -17.44 & -17.89 & -16 \\
32nd & -16.94 & -17.6 & -16.29 \\
38th & -16.44 & -17.5 & -15.39 \\
RVLPSS, \% & & & \\
20th & -17.54 & -18.01 & -17.07 \\
26th & -16.89 & -17.21 & -16.57 \\
32nd & -16.25 & -16.72 & -15.78 \\
38th & -15.61 & -16.37 & -14.85 \\
LALPSS, \% & & & \\
20th & 35.63 & 34.42 & 36.83 \\
26th & 34.05 & 33.21 & 34.89 \\
32nd & 32.48 & 31.25 & 33.72 \\
38th & 30.91 & 28.93 & 32.88 \\
RALPSS, \% & & & \\
20th & 36.21 & 35.37 & 37.05 \\
26th & 35.36 & 34.78 & 35.93 \\
32nd & 34.5 & 33.65 & 35.35 \\
38th & 33.64 & 32.27 & 35.01 \\
\hline
\end{tabular}

GA, gestational age; LV, left ventricular; RV, right ventricular; LA, left atrial; RA, left atrial; LPSS, global longitudinal peak systolic strain.

\section{Results}

This study included 101 healthy fetuses. The mean GA was $26.0 \pm 5.6$ weeks (range 16.3-38.6 weeks). The mean FHR was $143.8 \pm 11.3$ beats per minute (bpm), and temporal resolution was 26 frames per cycle (fpc).

In consequence of an incomplete view on LA or RA in some $4 \mathrm{CV}$, strain analysis was not feasible in $12(11.9 \%)$ cases for LA and 3 (3.0\%) cases for RA. As mentioned above, after inspection of model residuals, divergent cases were additionally excluded for strain analysis of LA in 3 (3.0\%) cases, RA in $4(4.0 \%)$ cases, and LV/RV ratio in 1 (1.0\%) case (Table 1). Descriptive statistics for LPSS values are accessible in Table 1. The mean values for LVLPSS and RVLPSS were $-17.44 \pm 2.29 \%$ and $-16.89 \pm 1.72 \%$. The mean values for LALPSS and RALPSS were $34.09 \pm 4.17 \%$ and $35.36 \pm 2.90 \%$. The mean LV/RV ratio was $1.03 \pm 0.15$.

Linear regression analysis revealed a significant association between GA and the LPSS for every cardiac cavity (Fig. 3). GA was positively correlated with LVLPSS $(b=$ $0.08 ; p<0.05)$ and RVLPSS $(b=0.10 ; p<0.05)$ and nega-

Atrial and Ventricular Strain Analysis in Normal Fetal Hearts
Table 3. Regression formula for correlation between LPSS of respective cardiac cavity and gestational age. $Z$-scores can be calculated for every measured strain value following the formula: $Z=[\mathrm{X}-\mathrm{M}(\mathrm{GA})] / \mathrm{SD}(\mathrm{GA})$

\begin{tabular}{ll}
\hline & Regression formula \\
\hline LVLPSS & $M(\mathrm{GA})=-19.618317+0.083628^{*} \mathrm{GA}(\mathrm{W})$ \\
& $\mathrm{SD}(\mathrm{GA})=2.246169$ \\
\hline RVLPSS & $M(\mathrm{GA})=-19.672545+0.106,851^{*} \mathrm{GA}(\mathrm{W})$ \\
& $\mathrm{SD}(\mathrm{GA})=1.61462281$ \\
\hline LALPSS & $M(\mathrm{GA})=40.865495-0.262013^{*} \mathrm{GA}(\mathrm{W})$ \\
& $\mathrm{SD}(\mathrm{GA})=-11.334207+1.072556^{*} \mathrm{GA}(\mathrm{W})-$ \\
\hline RALPSS & $0.017863^{*} \mathrm{GA}(\mathrm{W})$ \\
& $M(\mathrm{GA})=39.066751-0.142695^{*} \mathrm{GA}(\mathrm{W})$ \\
& $\mathrm{SD}(\mathrm{GA})=4.482349-0.067521^{*} \mathrm{GA}(\mathrm{W})$ \\
\hline
\end{tabular}

$\mathrm{M}(\mathrm{GA})$, mean value for the appropriate gestational age; $\mathrm{GA}(\mathrm{W})$, gestational age in exact weeks; SD(GA), standard deviation for the appropriate gestational age; $\mathrm{LV}$, left ventricular; $\mathrm{RV}$, right ventricular; LA, left atrial; RA, right atrial; LPSS, global longitudinal peak systolic strain.

Table 4. Indirect, direct, and total effect of the FHR on the relationship between GA and LVLPSS with 95\% confidence interval

\begin{tabular}{lccc}
\hline & Coefficient & \multicolumn{2}{l}{$95 \%$ confidence interval } \\
\cline { 3 - 4 } & & LLCI & ULCI \\
\hline Total effect & 0.084 & 0.0116 & 0.1557 \\
Direct effect & 0.110 & 0.0323 & $\begin{array}{c}0.1867 \\
\text { Indirect effect }\end{array}$ \\
\hline
\end{tabular}

LVLPSS, left ventricular global longitudinal peak systolic strain; GA, gestational age; LLCI, lower level confidence interval; ULCI, upper level confidence interval.

tively correlated with LALPSS $(b=-0.26 ; p<0.05)$ and RALPSS $(b=-0.14 ; p<0.05)$. There was no relevant effect of GA on the LV/RV ratio. From the 17th to 38th week of gestation, this means an absolute increase in LVLPSS by $1.68 \%$ and an absolute increase in RVLPSS by $2.1 \%$. Regarding atrial deformation, a total decrease in LALPSS by $5.46 \%$ and RALPSS by $2.94 \%$ was observed.

Marginal mean values for LPSS of every heart cavity for the 20th, 26th, 32nd, and 38th week of gestation are displayed in Table 2. The regression formula is shown in Table 3. Interactions between single LPSS values of different cardiac cavities did not correlate significantly. 
Table 5. Numerical results of BlandAltman analysis for intra- and interrater reliability with bias and lower and upper 95\% limit of agreement

\begin{tabular}{llllllll}
\hline & \multicolumn{2}{l}{ Intraobserver reliability } & & \multicolumn{3}{l}{ Interobserver reliability } \\
\cline { 2 - 4 } \cline { 7 - 8 } & bias & LLoA & ULoA & & bias & LLoA & ULoA \\
\hline LVLPSS & -0.06 & -1.36 & 1.23 & & -0.14 & -1.24 & 0.96 \\
RVLPSS & 0.03 & -1.27 & 1.34 & & -0.11 & -1.39 & 1.18 \\
LALPSS & 0.23 & -2.1 & 2.57 & & 0.24 & -3.01 & 3.48 \\
RALPSS & 0.21 & -2.27 & 2.69 & & -0.11 & -2.75 & 2.53 \\
\hline
\end{tabular}

LLoA, lower 95\% limit of agreement; ULoA, upper 95\% limit of agreement; LV, left ventricular; RV, right ventricular; LA, left atrial; RA, left atrial; LPSS, global longitudinal peak systolic strain.

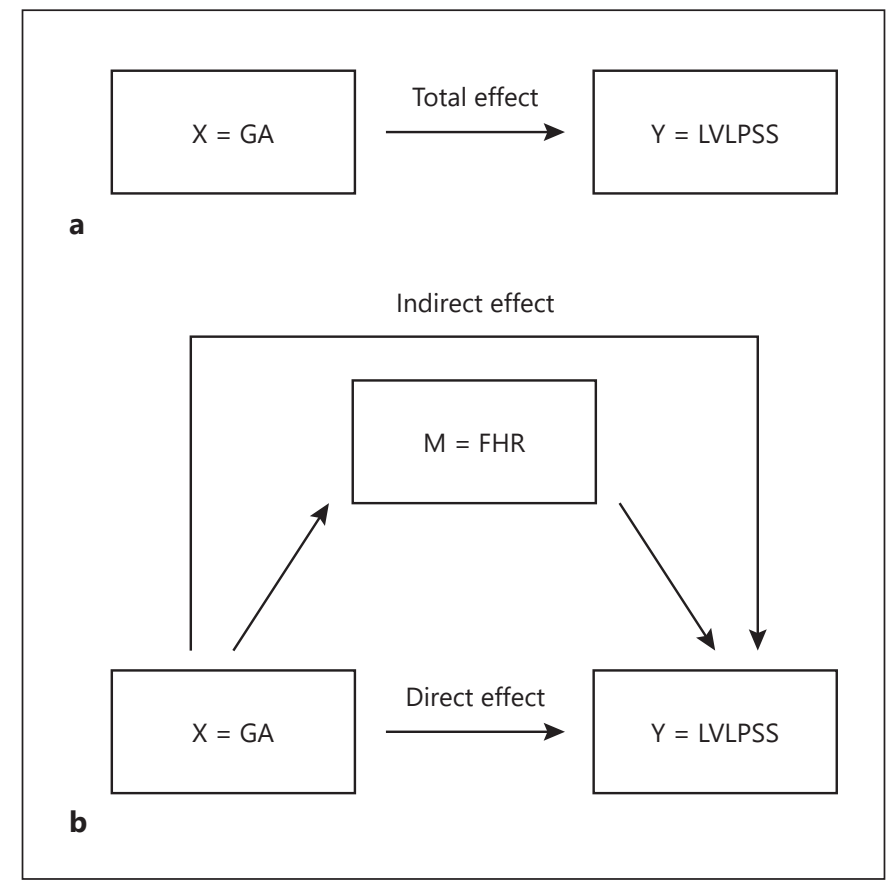

Fig. 4. Conceptual mediation model diagram for the relationship between LVLPSS, GA, and FHR: a Total effect of $\mathrm{X}=\mathrm{GA}$ on $\mathrm{Y}=$ LVLPSS, which is equivalent to the regression coefficient of linear regression analysis. This total effect could be decomposed in direct and indirect effects. b Direct effect of GA on LVLPSS, which describe correlation between GA and LVLPSS without the influence of FHR, and indirect effect of FHR on the association between GA and LVLPSS. The numerical results of the mediation analysis are shown in Table 3. GA, gestational age; LVLPSS, left ventricular global longitudinal peak systolic strain; FHR, fetal heart rate.

The effect of FHR on the association between GA and LVLPSS is displayed schematically in a conceptual diagram (Fig. 4) and numerically in Table 4. Using mediator analysis, association between GA and LVLPSS can be decomposed in different effects.
Without controlling for FHR, the linear association between GA and LVLPSS is positive and significant $(b=$ $0.08)$. When controlling for FHR, the association between GA and LVLPSS becomes stronger $(b=0.11)$. This can be explained by the mediating effect of the FHR: A higher GA leads to smaller FHR values. The FHR is associated positively with LVLPSS. Thus, it can be said that because GA is increasing and an increasing GA leads to decreasing FHR values, the LVLPSS values are decreasing with higher GA (indirect effect, $b=-0.03$ ). However, in addition to this negative effect of GA (via FHR) on LVLPSS, there is a stronger positive association between GA and LVLPSS, independent of the FHR $(b=0.11)$. Together, this negative indirect effect and the positive remaining association make the total effect between GA and LVLPSS $(b=0.08)$. From the 17th to 38th week of gestation, this means without the indirect effect of FHR, LVLPSS would absolutely increase by $2.31 \%$ instead of $1.68 \%$. The $95 \%$ confidence interval (BcA, 1,000 bootstrap repetitions) did not include zero; thus, the indirect effect can be interpreted as statistically significant. For strain analysis, Bland-Altman plots for intrarater reliability and interrater reliability are displayed in Figures 5 and 6 and Table 5 with 95\% limits of agreement.

\section{Discussion}

The objective of this study was to examine ventricular and atrial MF in fetal cardiac 4CV using 2D-STE. Investigation of atrial and ventricular LPSS was feasible, and a correlation between GA and LPSS for every cardiac cavity was detectable. The FHR turned out to be an effector for the association between GA and LPSS. This study is one of the 1st attempts to measure atrial LPSS.

Ventricular strain values are well established and reported with acceptable reproducibility [5-12]. However, small differences in ventricular LPSS values are notice- 

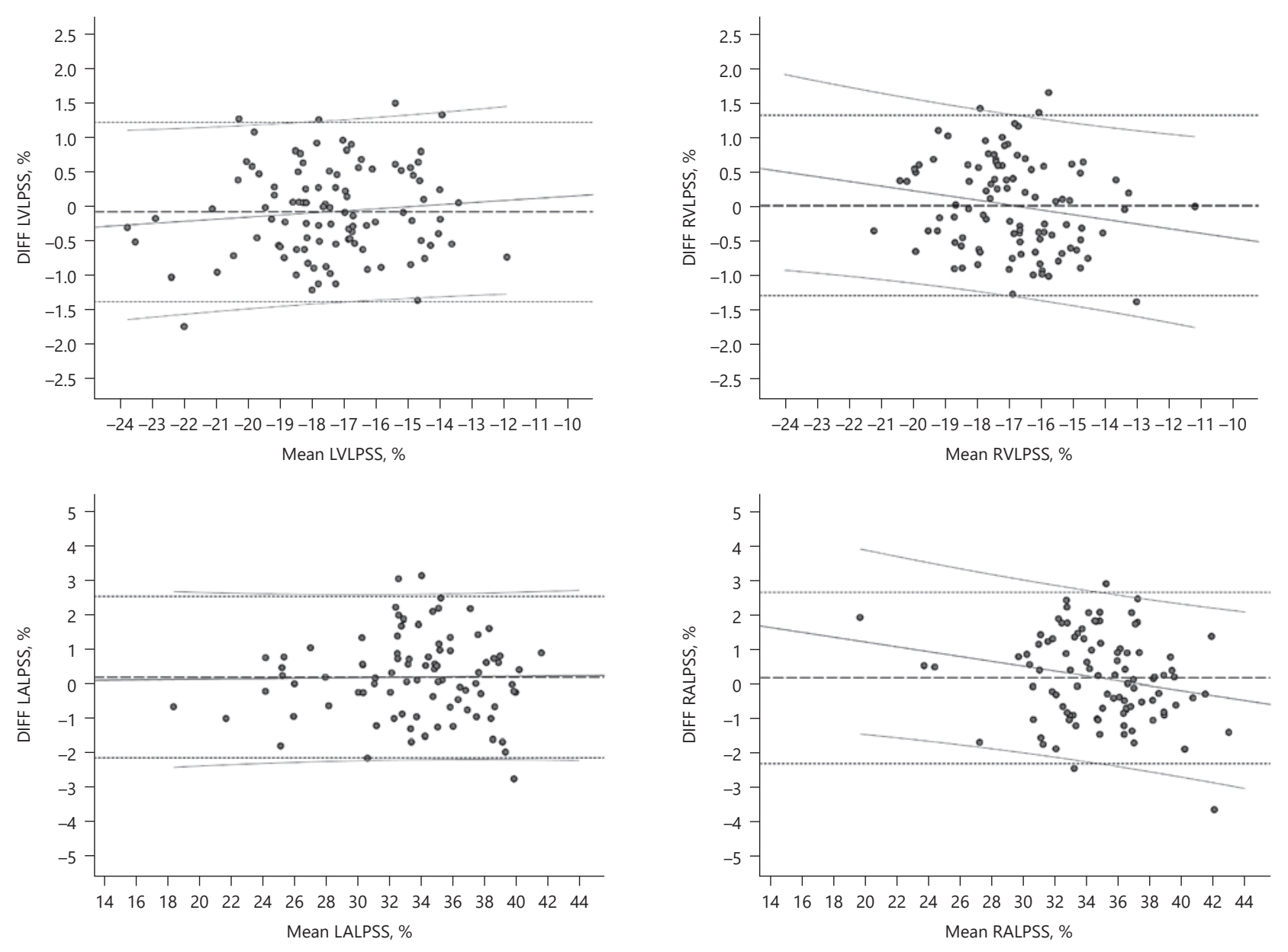

Fig. 5. Bland-Altman plots for intrarater reliability for measurements of every cardiac cavity with mean and $95 \%$ limits of agreement (dotted horizontal lines). In addition, regression of the differences of mean values with $95 \%$ confidence interval is shown (gray solid lines).

able compared with previous studies of Enzensberger et al. [10], what might be the result of different speckle tracking software packages [10-12]. Our own data revealed that the variability in image acquisition, the optimal temporal resolution, and the use of different ultrasound packages are important influencing factors [1012]. Definitions for a common standard for 2D-STE were published to standardize deformation imaging in adult cardiology [27]. A widespread ranging between actual fetal global longitudinal strain values is still noticeable, which might be explained by different methods and technical conditions for 2D-STE (Tables 6, 7). Hence, more detailed standardization of image acquisition and 2D speckle tracking, especially for fetal echocardiography, seems to be more and more important for future research.
These technical challenges were even more important for atrial deformation analysis. In consequence of incomplete view on the left and right myocardium, strain analysis was not feasible in $12(11.9 \%)$ cases for LA and $3(3 \%)$ cases for RA. In order to further increase the feasibility, an adaption of the $4 \mathrm{CV}$ with regard to an optimized atrial view would be necessary. Maximal resolution during image acquisition was necessary to minimize this dropout rate by displaying the thin atrial myocardium in full thickness. However, Bland-Altman analysis shows marginal bias and mean difference in intra- and interobserver reliability for every cardiac cavity.

Until now, only Dahlbäck et al. [18] performed atrial 2D-STE in normal fetal hearts and revealed a left atrial global longitudinal strain of $23.2 \pm 13.1 \%$ and right atrial 

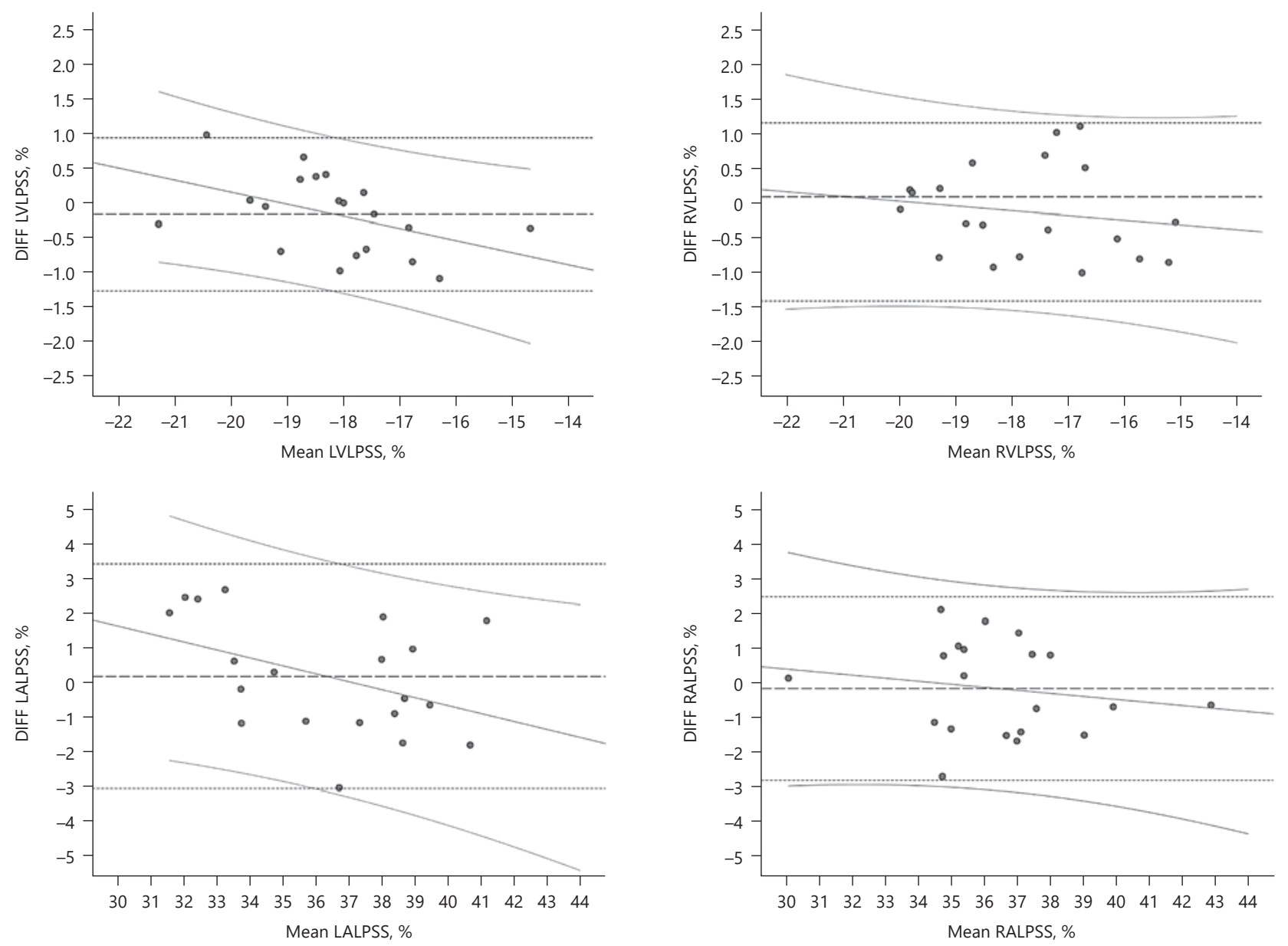

Fig. 6. Bland-Altman plots for interrater reliability for measurements of every cardiac cavity with mean and $95 \%$ limits of agreement (dotted horizontal lines). In addition, regression of the differences of mean values with $95 \%$ confidence interval is shown (gray solid lines).

global longitudinal strain of $33.6 \pm 19.7 \%$. The authors used a different speckle tracking pattern, which exclude the interatrial septum from strain analysis. High variability and low ICC values for global longitudinal strain as well as the differences in LALPSS values might be caused by this different speckle tracking pattern. Steinhard et al. [19] recently reported atrial strain analysis using tissue Doppler imaging (TDI).

Our study has strengths and limitations. To perform atrial strain analysis, the use of an STE software package, which is also used in adult cardiology, was necessary. A possible influence of the used software package cannot be excluded. Also, the frame rate in STE is a part of discussion in the current research. For fetal STE, a frame rate of at least $80 \mathrm{fps}$ is recommended by DeVore et al. [33], which results in a temporal resolution of $40 \mathrm{fpc}$. However, a consensus about the optimal frame rate and temporal resolution is still missing. We generally agree that using high frame rates might have an influence on STE, especially regarding the exact definition of the end systole. However, further studies should consider a possible intervendor variability in technical basics of different software packages. A detailed knowledge about the use of radiofrequency-STE and grayscale-STE in the respective software packages is needed to assess the influence of frame rate correctly. Also, the usage of the same frame rate in every clip could be an important factor for strain analysis. The use of $26 \mathrm{fpc}$ in this study should be considered as strain values could be underestimated. A part from these abovementioned technical challenges, other limita- 
Table 6. Published global longitudinal peak systolic strain values for the left ventricle

\begin{tabular}{|c|c|c|c|c|c|c|}
\hline Patey et al. [13] & 2017 & 108 & $39 \pm 1.5$ & Toshiba & -11.0 & 4.0 \\
\hline Ishii et al. [8] & 2012 & 81 & $19-42$ & Siemens & -15.2 & 2.7 \\
\hline Miranda et al. [28] & 2017 & 12 & $19-33$ & Toshiba & -16.7 & NR \\
\hline Enzensberger et al. [10] & 2017 & 101 & $17-39$ & Toshiba & -17.5 & NR \\
\hline Germanakis et al. [30] & 2012 & 144 & $14-39$ & Siemens & -21.9 & 3.7 \\
\hline DeVore et al. [14] & 2018 & 200 & $20-40$ & GE & -22.9 & 3.5 \\
\hline Kapusta et al. [15] & 2013 & 44 & $30-34$ & GE & -24.7 & 4.8 \\
\hline Kapusta et al. [9] & 2012 & 78 & $20-24$ & GE & -24.9 & 4.6 \\
\hline Di Salvo et al. [31] & 2008 & 100 & $20-32$ & $\mathrm{GE}$ & -25.0 & 4.0 \\
\hline Willruth et al. [32] & 2011 & 150 & $13-39$ & Siemens & -27.9 & 10.5 \\
\hline
\end{tabular}

LV, left ventricular; SD, standard deviation; NR, not reported.

Table 7. Published global longitudinal peak systolic strain values for the right ventricle

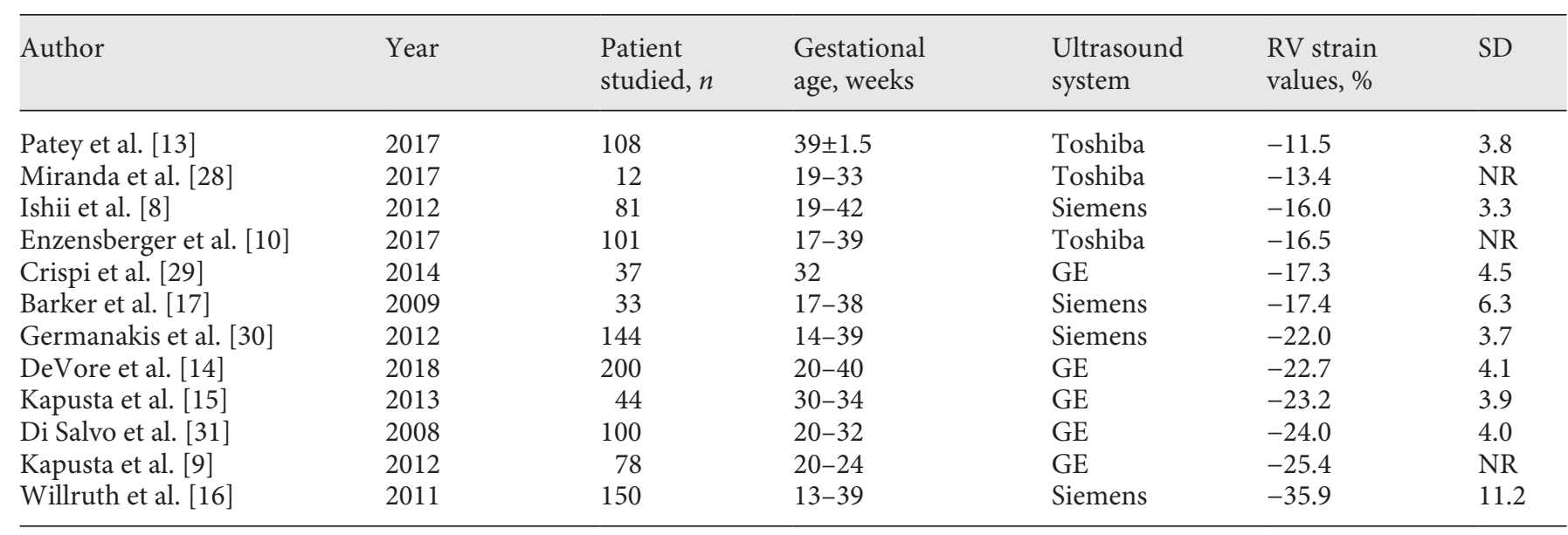

$\mathrm{RV}$, right ventricular; SD, standard deviation; $\mathrm{NR}$, not reported.

tions should be emphasized. In accordance with Koca et al. [20], we include the interatrial septum for better orientation in strain analysis. From our point of view, using valve level as orientation in strain analysis is the best way to guarantee consistent results in 2D-STE. Also, technical conditions of $2 \mathrm{D}-\mathrm{CPA}$ require clear orientation points like the mitral plane. The impact of open foramen ovale should be regarded at this point. By including the interatrial septum in the strain analysis, we accept a change of LPSS values for better reproducibility of 2D-STE, which is even reached with a bias of smaller than 1.0 in BlandAltman analysis. Furthermore, a two-dimensional section of the atria at cardiac $4 \mathrm{CV}$ likely does not reflect the maximum diameter of the fetal atria. To assess the true volume change, three-dimensional STE seems to be a solution. However, fetal cardiac $4 \mathrm{CV}$ is a part of the basic ultrasonic screening and might be more suitable for $2 \mathrm{D}$ STE and a clinical implementation of it. A methodology for atrial strain analysis, which is appliable in clinical routine, was one of the major ideas of this study. This could be reached with a good reproducibility.

In addition, linear regression analysis revealed significant correlations between GA and LPSS for every cardiac cavity. Considering different contraction directions related to the valve level, atrial and ventricular LPSS have different algebraic signs. Consequently, absolute values of 


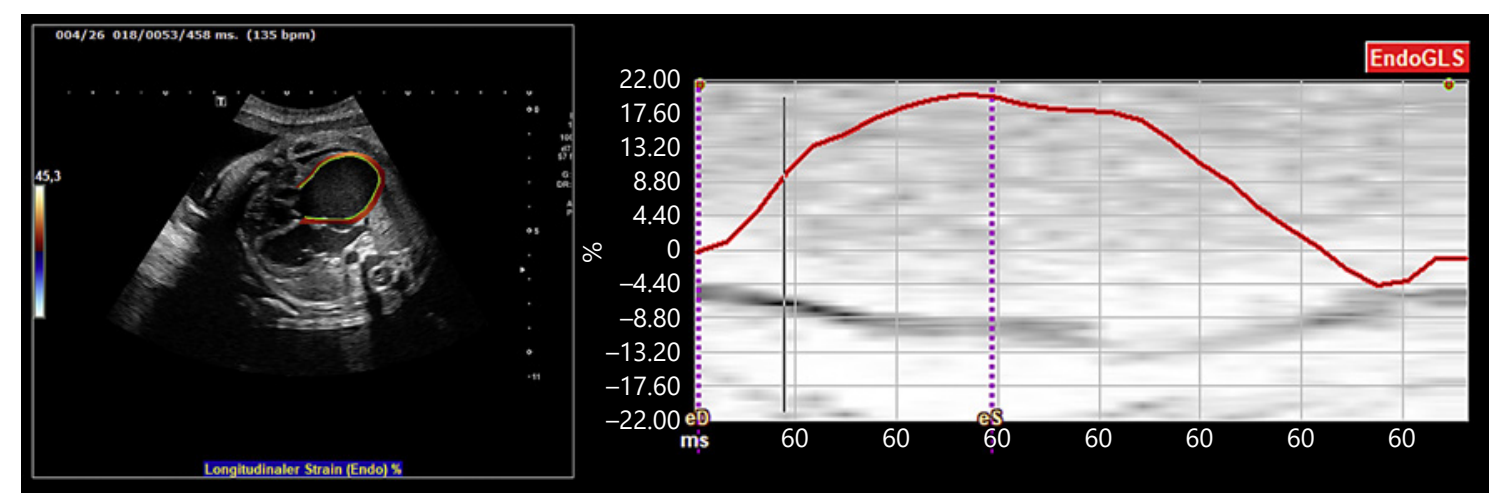

Fig. 7. Atrial strain analysis in a fetus with PAIVS. Left: $4 \mathrm{CV}$ of a fetus with PAIVS with myocardial border tracking of the right atrium. Right: atrial global longitudinal peak systolic strain measurement within 1 cardiac cycle. PAIVS, pulmonary atresia with intact ventricular septum; 4CV, 4-chamber view.

LPSS reflect the magnitude of deformation irrespective of its direction. This means that the positive correlations between GA and ventricular LPSS values as well as the negative correlations between GA and atrial LPSS can be interpreted as a phenomenological decrease in myocardial strain between the 17th and 38th week of gestation. As mentioned by Willruth et al. [16], a possible reason for this decrease in myocardial strain might be an increase in cardiac afterload caused by changes of placental resistance during gestation $[16,34,35]$. The meaning of GA as an influencing factor for strain analysis has to be examined in further prospective studies. Longitudinal examination of the same fetus in further studies seems to be adequate to confirm the results of linear regression analysis.

Interactions between single LPSS values of different cardiac cavities did not correlate significantly. However, this stands in contrast to actual MRI studies in pediatric population, which show an atrioventricular interaction [36]. Further studies need to verify this correlation, which might be the result of the relatively small study population.

To examine possible influencing factors for the result of linear regression analysis, we perform a mediator analysis exemplary for left ventricular strain values. Interpreting mediator analysis, we show a significantly indirect effect of FHR on the relationship between LVLPSS and GA. A clearer decrease in myocardial strain during gestation can be assumed without the indirect effect of FHR. This result seems to be plausible because myocardial shortening will be more effective with decrease in FHR. Besides the changes of placental resistance as a possible reason for the dependency between LPSS and GA, a decrease in the FHR during gestation might partially compensate the phenomenologically shrinking LPSS during gestation to guarantee cardiac output. High confidence intervals for indirect effect might be the result of relatively low numbers of incoming $4 \mathrm{CV}$. Future studies should clarify this effect of the FHR for all cardiac cavities. Other influencing factors such as heart volume or stroke volume will be examined to explain the total effect of GA on LPSS, whose magnitude is mainly caused by the direct effect.

Finally, the potential and applicability of especially atrial strain measurements in fetuses at risk should be demonstrated. By performing atrial strain analysis in a fetal heart with pulmonary atresia with intact ventricular septum (PAIVS), we could reveal a strong reduction in RALPSS compared with our control group, reflecting reduced right ventricular MF in this case (Fig. 7). The RALPSS in the fetus with PAIVS was $20.65 \%$. An evaluation of atrial deformation for fetuses at risk seems to be generally possible. It should be noticed that our method, which includes interatrial septum in strain analysis, seems to make atrial deformation analysis feasible. Furthermore, Koca et al. [20] demonstrated the potential of atrial strain analysis by using atrial LPSS as a predictor for the success of therapeutic interventions, which also may be applied in prenatal medicine.

In conclusion, quantification of MF using 2D-STE for both atrial and ventricular deformation analysis was feasible in cardiac $4 \mathrm{CV}$. We present in this study atrial LPSS values, which enable further research about the relevance of the atriums for pathological conditions prenatally. Furthermore, GA-dependent LPSS values could be determined for every cardiac cavity. For future research on MF of the fetus, considering GA as an influencing factor for deformation analysis seems to be adequate. 


\section{Acknowledgements}

Technical support was provided by TomTec Imaging Systems GmbH, Unterschleissheim, Germany.

\section{Statement of Ethics}

This study complies with the guidelines for human studies and was conducted ethically in accordance with the World Medical Association Declaration of Helsinki. The institutional ethical review board of Justus Liebig University Giessen approved this study (ethical approval number: AZ 186/18). Informed written consent was obtained from all participants.

\section{Disclosure Statement}

The authors were supported by TomTec Imaging Systems $\mathrm{GmbH}$, Unterschleissheim, Germany, with the commercially available software TomTec Image Arena.

\section{Funding Sources}

There are no sources of funding.

\section{Author Contributions}

Every named author was involved in data collection, evaluation of the data, and the writing process of this manuscript.

\section{References}

1 Van Mieghem T, Giusca S, DeKoninck P, Gucciardo L, Doné E, Hindryckx A, et al. Prospective assessment of fetal cardiac function with speckle tracking in healthy fetuses and recipient fetuses of twin-to-twin transfusion syndrome. J Am Soc Echocardiogr. 2010 Mar; 23(3):301-8.

2 DeKoninck P, D'hooge J, Van Mieghem T, Richter J, Deprest J. Speckle tracking echocardiography in fetuses diagnosed with congenital diaphragmatic hernia. Prenat Diagn. 2014; 34(13):1262-7.

3 Patey O, Carvalho JS, Thilaganathan B. Perinatal changes in fetal cardiac geometry and function in gestational diabetic pregnancies at term: perinatal fetal cardiac dysfunction in GDM. Ultrasound Obstet Gynecol. 2018 Dec; 54(5):634-42.

4 Cohen J, Binka E, Woldu K, Levasseur S, Glickstein J, Freud LR, et al. Myocardial strain abnormalities in fetuses with pulmonary atresia and intact ventricular septum. Ultrasound Obstet Gynecol. 2019 Apr;53(4):512-9.

5 Amundsen BH, Helle-Valle T, Edvardsen T, Torp H, Crosby J, Lyseggen E, et al. Noninvasive myocardial strain measurement by speckle tracking echocardiography: validation against sonomicrometry and tagged magnetic resonance imaging. J Am Coll Cardiol. 2006 Feb;47(4):789-93.

6 Younoszai AK, Saudek DE, Emery SP, Thomas JD. Evaluation of myocardial mechanics in the fetus by velocity vector imaging. J Am Soc Echocardiogr. 2008 May;21(5):470-4.

7 Matsui H, Germanakis I, Kulinskaya E, Gardiner HM. Temporal and spatial performance of vector velocity imaging in the human fetal heart. Ultrasound Obstet Gynecol. 2011 Feb; 37(2):150-7.

8 Ishii T, McElhinney DB, Harrild DM, Marcus EN, Sahn DJ, Truong U, et al. Circumferential and longitudinal ventricular strain in the normal human fetus. J Am Soc Echocardiogr. 2012 Jan;25(1):105-11.
9 Kapusta L, Mainzer G, Weiner Z, Deutsch L, Khoury A, Haddad S, et al. Second trimester ultrasound: reference values for two-dimensional speckle tracking-derived longitudinal strain, strain rate and time to peak deformation of the fetal heart. J Am Soc Echocardiogr. 2012 Dec;25(12):1333-41.

10 Enzensberger C, Achterberg F, Graupner O, Wolter A, Herrmann J, Axt-Fliedner R. Wallmotion tracking in fetal echocardiographyInfluence of frame rate on longitudinal strain analysis assessed by two-dimensional speckle tracking. Echocardiography. 2017 Jun;34(6): 898-905.

11 Enzensberger C, Achterberg F, Degenhardt J, Wolter A, Graupner O, Herrmann J, et al. Feasibility and reproducibility of two-dimensional wall motion tracking (WMT) in fetal echocardiography. Ultrasound Int Open. 2017 Feb;3(1):E26-33.

12 Enzensberger C, Rostock L, Graupner O, Götte M, Wolter A, Vorisek C, et al. Wall motion tracking in fetal echocardiography: application of low and high frame rates for strain analysis. Echocardiography. 2019 Feb;36(2): 386-93.

13 Patey O, Gatzoulis MA, Thilaganathan B, Carvalho JS. Perinatal changes in fetal ventricular geometry, myocardial performance, and cardiac function in normal term pregnancies. J Am Soc Echocardiogr. 2017 May; 30(5):485-e5.

14 DeVore GR, Klas B, Satou G, Sklansky M. Longitudinal annular systolic displacement compared to global strain in normal fetal hearts and those with cardiac abnormalities. J Ultrasound Med. 2018;37(5):1159-71.

15 Kapusta L, Mainzer G, Weiner Z, Deutsch L, Khoury A, Haddad S, et al. Changes in fetal left and right ventricular strain mechanics during normal pregnancy. J Am Soc Echocardiogr. 2013 Oct;26(10):1193-200.
16 Willruth AM, Geipel AK, Fimmers R, Gembruch UG. Assessment of right ventricular global and regional longitudinal peak systolic strain, strain rate and velocity in healthy fetuses and impact of gestational age using a novel speckle/feature-tracking based algorithm. Ultrasound Obstet Gynecol. 2011; 37(2):143-9.

17 Barker PC, Houle H, Li JS, Miller S, Herlong JR, Camitta MG. Global longitudinal cardiac strain and strain rate for assessment of fetal cardiac function: novel experience with velocity vector imaging. Echocardiography. 2009; 26(1):28-36.

18 Dahlbäck C, Gudmundsson S. Investigations on atrial function in fetuses with signs of impaired placental function. Prenat Diagn. 2015; 35(6):605-11.

19 Steinhard J, Entenmann A, van der Valk E, Schmitz R, Heinig J, Laser KT, et al. The transverse four-chamber view for the assessment of atrial tissue deformation in the fetus. PloS One. 2018 Jul;13(7):e0199581.

20 Koca H, Demirtas AO, Kaypaklı O, Icen YK, Sahin DY, Koca F, et al. Decreased left atrial global longitudinal strain predicts the risk of atrial fibrillation recurrence after cryoablation in paroxysmal atrial fibrillation. J Interv Card Electrophysiol. 2019 Jun;58(1):51-9.

21 Zaidi SJ, Lefaiver CA, Muangmingsuk S, Cui VW, Roberson DA, Penk J. Right ventricular longitudinal shortening before and after stage I surgical palliation correlates with outcomes. Pediatr Cardiol. 2018 Mar;39(3):526-32.

22 Zaidi SJ, Penk J, Cui VW, Kanjanauthai S, Roberson DA. Right ventricular systolic function parameters in hypoplastic left heart syndrome. Pediatr Cardiol. 2018 Oct;39(7): 1423-32.

23 Kawagishi T. Speckle tracking for assessment of cardiac motion and dyssynchrony. Echocardiography. 2008;25(10):1167-71.

24 Acock AC. A gentle introduction to Stata, 2nd ed. College Station: Stata Press; 2016. 
25 Kurmanavicius J, Wright EM, Royston P, Wisser J, Huch R, Huch A, et al. Fetal ultrasound biometry: 1 . Head reference values. $\mathrm{Br}$ J Obstet Gynaecol. 1999;106(2):126-35.

26 Hayes AF. Introduction to mediation, moderation, and conditional process analysis: a regression-based approach, 2nd ed. New York: Guilford Press; 2018.

27 Voigt JU, Pedrizzetti G, Lysyansky P, Marwick TH, Houle H, Baumann R, et al. Definitions for a common standard for 2D speckle tracking echocardiography: consensus document of the EACVI/ASE/industry task force to standardize deformation imaging. J Am Soc Echocardiogr. 2015 Feb;28(2):183-93.

28 Miranda JO, Hunter L, Tibby S, Sharland G, Miller O, Simpson JM. Myocardial deformation in fetuses with coarctation of the aorta: a case-control study. Ultrasound Obstet Gynecol. 2017;49(5):623-9.
29 Crispi F, Bijnens B, Sepulveda-Swatson E, Cruz-Lemini M, Rojas-Benavente J, Gonzalez-Tendero A, et al. Postsystolic shortening by myocardial deformation imaging as a sign of cardiac adaptation to pressure overload in fetal growth restriction. Circ Cardiovasc Imaging. 2014 Sep;7(5):781-7.

30 Germanakis I, Matsui H, Gardiner HM. Myocardial strain abnormalities in fetal congenital heart disease assessed by speckle tracking echocardiography. Fetal Diagn Ther. 2012; 32(1-2):123-30.

31 Di Salvo G, Russo MG, Paladini D, Felicetti M, Castaldi B, Tartaglione A, et al. Two-dimensional strain to assess regional left and right ventricular longitudinal function in 100 normal foetuses. Eur J Echocardiogr. 2008 May;9(6):754-6.
32 Willruth AM, Geipel A, Berg C, Fimmers R, Gembruch U. Assessment of left ventricular global and regional longitudinal peak systolic strain, strain rate and velocity with feature tracking in healthy fetuses. Ultraschall Med. 2011 Feb;33(7):E293-8.

33 DeVore GR, Polanco B, Satou G, Sklansky M. Two-dimensional speckle tracking of the fetal heart: a practical step-by-step approach for the fetal sonologist. J Ultrasound Med. 2016; 35(8):1765-81.

34 Gardiner HM. Response of the fetal heart to changes in load: from hyperplasia to heart failure. Heart. 2005 Jul;91(7):871-3.

35 Kiserud T, Acharya G. The fetal circulation. Prenat Diagn. 2004;24(13):1049-59.

36 Riesenkampff E, Mengelkamp L, Mueller M, Kropf S, Abdul-Khaliq H, Sarikouch S, et al. Integrated analysis of atrioventricular interactions in tetralogy of Fallot. Am J Physiol Heart Circ Physiol 2010 Aug;299(2):H364-71. 